\title{
Conidiobolus coranatus infection of the para-nasal sinuses masquerading as a sino-nasal tumour
}

\author{
M M A Jayawickrama ${ }^{1}$, H U P Gamage ${ }^{2}$, P D Perera ${ }^{3}$ \\ Ceylon Medical Journal 2012; 57: 87-88
}

\section{Introduction}

Fungal infections of the para nasal sinuses are uncommon [1, 2]. We report a rare case of Conidiobolus coronatus infection of the para-nasal sinuses in a 32-year old immuno competent male presenting as a sino-nasal tumour extending to the brain which completely resolved following resection and anti fungal therapy. Nasal sinus infection due to $C$. coranatus has not been reported previously in Sri Lanka.

\section{Case report}

A 32-year old man working in a hardware store presented with right nasal obstruction, nasal discharge and frequent sneezing of three months duration. He had no other significant medical illness. Examination revealed enlarged right turbinate obstructing the nasal cavity and fullness over the maxillary area. CT scan of the para-nasal sinuses revealed an extensive soft tissue growth in the right maxillary antrum, involving both ethmoids and sphenoid sinuses with erosion of the cribriform plate with extension into the frontal lobe of the brain. A biopsy was taken under anaesthesia by the infra turbinate route and sent for histology. Heamatoxylin and eosin (H\&E) stained sections revealed fibro connective tissue showing extensive areas of necrosis with eosinophil infiltration and eosinophilic vasculitis. There was no evidence of a neoplasm in multiple sections examined. A possibility of Wagener's granulomatosis was suggested. Anti neutrophilic cytoplasmic antibody to cytoplasm and perinuclear antigen were negative. A second biopsy was taken and a piece was sent to the Medical Research Institute for mycological studies. C. coronatus / Entomophth oraceous fungus was isolated on direct microscopy and culture. $\mathrm{H} \& \mathrm{E}$ stained sections revealed granulomas with foreign body type multinucleated giant cells, chronic inflammation with abundant oesinophils. Periodic acid schiff and Grocotts stain revealed fungal filaments. Right lateral rhinotomy and medial maxillectomy was done. The patient was treated with potassium iodide (KI), ithaconazole and cotrimoxazole for six months. Liver function tests, blood urea and creatinine was monitored every two weeks. After four weeks there was an increase in alanine transaminase enzymes (ALT) and KI dose was adjusted to maintain the ALT level within the normal range. CT scan was done two months after commencing therapy and compared with the initial films and showed a marked reduction in soft tissue swelling of the nasal cavity, ethmoids, maxillary and sphenoid sinuses, indicating a good response to anti fungal therapy. There was mucosal thickening in all the sinuses but no soft tissue growth in the nasal cavity or erosion of the cribriform plates. Four months later right rigid nasal endoscopy revealed nasal adhesions. Nasal septoplasty with submucous resection was done. CT scan six months after initial presentation showed complete clearance of the sinuses. The patient was free of recurrence 60 months later.

\section{Discussion}

Invasive paranasal sinus fungal infections are mostly described in South East Asian and Chinese people and occur in immunocompetant hosts [1-3]. Intra cranial extension of sinus infection with bone erosion is described in aspergillosis and mucormycosis with a very good response to anti fungal treatment [4,5]. Intra cranial extension has not been previously reported in C. coranatus infection [1]. Radiological imaging of chronic sinusitis due to fungi sometimes mimic a tumour with bone erosion and intracranial spread [6]. Infection with C. coronatus causes chronic granulomatous lesions of the nasal sinuses spreading to contiguous skin and invading blood vessels. Bone erosion mimics a malignant tumour. This fungus is also called Entomophthora mycosis, Conidibolus mycosis, Condidibolus and Rhinoentomopth thromycosis [6]. This infection affects healthy adult males but is also reported in immuno compromised individuals [7]. Patients commonly present with chronic sinusitis and nasal obstruction often mimicking a sino-nasal tumour. The mode of transmission is inhalation of fungal spores.

C. coranatus has a universal, mainly tropical distribution. It is a saprophyte in soil and on decaying vegetation. It has been isolated in tropical rain forest of Asia, insects and in dolphins. This fungus grown on culture media produce villous conidia giving a lace like

Departments of ${ }^{1}$ Pathology, ${ }^{2} E N T$ Surgery, Provincial Hospital Ratnapura and ${ }^{3}$ Department of Mycology, Medical Research Institute, Colombo, Sri Lanka.

Correspondence: MMAJ, e-mail: <modinij@yahoo.com>. Received 29 August and revised version accepted 22 October 2012. Competing interests: none declared. 
appearance. Colonies grow rapidly and are flat, cream coloured and become radially folded covered by a fine powdery white surface consisting of mycelium and condiospores. Tan coloured colonies become brown with age [7].

Treatment consists of a combination of three or more drugs and surgery. KI, amphoteracine B, ketaconazole and itraconazole [1]. Our patient had complete clearance of the nasal sinuses on CT scan 6 months after the initial presentation and was free of recurrence 72 months later on follow up. Long term follow up is required as recurrences are common.

\section{References}

1. Thammayya A. Zygomycosis due to Conidiobolus coronatus in West Bengal. Indian Journal of Chest Disease Allied Science 2000; 4: 305-9.

2. Manosuthi W, Sungkanuparph S, Hongmance P, et al.
Concurrent chromomycosis and aspergillosis of the maxillary sinus. Journal of Medical Association of Thailand 2004; 9: 1112-5.

3. Scharf JL, Soliman AM. Chronic Rhizopus invasive fungal rhino sinusitis in an immunocompetant host. Laryngoscope 2004; 9: 1533-5.

4. Yamanoi T, Shibano K, Soeda T, et al. Intracranial invasive aspergillosis originating in the sphenoid sinus: a successful treatment with high dose itraconazole in three cases. Tohoku Journal of Experimental Medicine 2004; 203: 133-9

5. Brun AL, Guichard JP, Assouline E, et al. Rhinocerebral mucormycosis. Journal of Neuroradiology 2004; 31: 74-6

6. Moulin G, Pascal T, Jacquier A, et al. Radiological imaging of chronic sinusitis in the adult. Journal of Radiology 2003; 84: 901-19.

7. Ajello L, Hay RJ, in Topley and Wilson's Microbiology and Microbial Infection edited by Collia L, Balow A, Sussman M. 9th Edition. Volume 4, 1998 Arnold; London 253-6. 